\title{
Acyloxyacyl Hydrolase Modulates the Gut Microbiome Through Transcriptional Regulators of Corticotropin-Releasing Factor
} \author{
Schaeffer', and David J. Klumpp ${ }^{1,3+}$ \\ Department of Urology ${ }^{1}$ \\ Division of Thoracic Surgery² \\ Department of Microbiology-Immunology \\ Feinberg School of Medicine \\ Northwestern University, Chicago, IL
}

Afrida Rahman-Enyart, Lizath M. Aguiniga', Wenbin Yang², Ryan E. Yaggie',

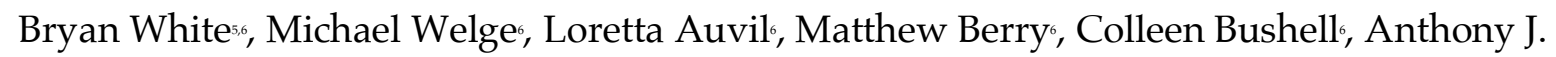

School of Pharmacy 4

University of Southern California, Los Angeles, CA

Department of Animal Sciences

National Center for Supercomputing Applications ${ }^{6}$

University of Illinois at Urbana-Champaign, Urbana, IL

+Address all correspondence to

d-klumpp@northwestern.edu

16-719 Tarry Building

303 East Chicago Avenue, Chicago, IL

312.908.1996 P

312.908.7275 F

The authors have declared that no conflicts of interest exist 


\section{ABSTRACT}

Gut microbiome-host interactions play a crucial role in health and disease. Altered gut microbiome composition has been observed in patients with interstitial cystitis/bladder pain syndrome (IC/BPS), a disorder characterized by pelvic pain, voiding dysfunction, and often comorbid with anxiety/depression. We recently showed that mice deficient for acyloxyacyl hydrolase $(\mathrm{AOAH})$ mimic pelvic pain symptoms and comorbidities of IC/BPS and also exhibit gut dysbiosis. In addition, we previously identified that the conditional knockout (cKO) of two transcriptional regulators of the gene encoding corticotropin-releasing factor, $\mathrm{Crf}$, that are downstream of $\mathrm{AOAH}$, aryl hydrocarbon receptor $(\mathrm{AhR})$ and peroxisome proliferator-activated receptor- $\gamma(\operatorname{PPAR} \gamma)$, alleviate anxiety/depressive and voiding phenotypes of AOAH-deficient mice. Here, we examined the effects of AhR and PPAR $\gamma$ in CRF-expressing cells on the dysbiosis of AOAH-deficiency. AOAH-deficient mice with cKO of PPAR $\gamma$ and AhR/PPAR $\gamma$ exhibited reduced pelvic allodynia compared to AOAH-deficient mice, suggesting a role for PPAR $\gamma$ in regulating pelvic pain. $16 \mathrm{~S}$ rRNA sequencing of fecal stool from female AOAH-deficient mice with a cKO of AhR and/or PPAR $\gamma$ in CRF-expressing cells identified altered gut microbiota distinct from AOAH-deficient stool. The $\mathrm{CKO}$ of AhR and PPAR $\gamma$ showed improved cecum barrier function in females compared to AOAH-deficient mice, whereas males were primarily affected by $\operatorname{PPAR} \gamma$, suggesting sex differences in gut responses. Pair-wise comparison of microbiota also suggested sex differences in response to AOAH-deficiency and conditional knockout of $A h R$ and $P P A R \gamma$. Our findings suggest that the dysbiosis and leaky gut of AOAH deficiency is mediated by AhR and PPAR $\gamma$ in CRF-expressing cells and reveal a novel mechanism and therapeutic targets for pelvic pain. 


\section{INTRODUCTION}

The gut microbiome plays an important role in health and metabolism, and dysbiosis has been associated with numerous diseases and visceral pain $(7,25,30)$. It has previously been shown that patients suffering from interstitial cystitis / bladder pain syndrome (IC/ BPS or "IC"), a chronic condition characterized by pelvic pain and urinary dysfunction, have altered fecal microbiota $(3,4,14,21)$. IC/BPS continues to be a clinical challenge due to its unknown etiology and lack of effective biomarkers (21). With this goal in mind, we previously conducted a genetic screen to identify modulators of pelvic pain severity and identified a locus encoding acyloxyacyl hydrolase (Aoah) as a candidate gene (37).

AOAH is a neutrophil lipase best known for its role in the detoxification of bacterial lipopolysaccharides (LPS) through the removal of secondary acyl chains from the lipid A moiety, resulting in the downregulation of host response to bacterial infection and the attenuation of the inflammatory response $(10,12,18,22,31)$. We have previously demonstrated that AOAHdeficient mice develop a phenotype of spontaneous pelvic pain and are more susceptible to induced pelvic pain models (37). In addition, AOAH-deficient mice mimic key aspects of IC/BPS, including gut dysbiosis and several associated comorbidities (1, 2, 26, 37). Altering the gut microbiome of AOAH-deficient mice by co-housing or gavage of healthy stool slurry alleviates pelvic pain, suggesting a role for gut flora in modulating the pelvic pain phenotype (26).

Corticotropin-releasing factor $(\mathrm{CRF})$ is a well-known regulator of the stress response through the regulation of the hypothalamic-pituitary-adrenal (HPA) axis and is also a key modulator in pain responses. IC/BPS patients exhibit altered diurnal cortisol expression implicating HPA axis dysregulation (19). Previous studies in our lab demonstrated that AOAHdeficient mice exhibit increased expression of central nervous system (CNS) arachidonic acid, increased Crf expression in the paraventricular nucleus, and elevated serum corticosterone, consistent with dysregulation of the HPA axis (2). In addition, we identified two arachidonic aciddependent transcription regulators of $\mathrm{Crf}$ that play a role in AOAH-deficient phenotypes, aryl 
hydrocarbon receptor $(\mathrm{AhR})$ and peroxisome proliferator-activated receptor- $\gamma$ (PPAR $\gamma$ ) (2). Conditional knockout (cKO) of $A h R$ and/or PPAR $\gamma$ in CRF-expressing cells in AOAH-deficient mice rescued phenotypes often observed in IC/BPS patients, such as depressive behavior and voiding dysfunction $(1,2)$.

CRF also plays a role in regulating the complex communication pathways of the microbiota-gut-brain axis and alterations of CRF expression have been shown to promote changes in gut microbiota composition (16). Since we have observed gut dysbiosis in AOAH-deficient mice and a role for CRF in AOAH-deficient phenotypes, we hypothesized that the cKO of transcriptional regulators of Crf would alter gut dysbiosis mediated by AOAH deficiency. We observed that the cKO of $A h R$ and/or PPAR $\gamma$ in CRF-expressing cells in AOAH-deficient mice altered the gut microbiome and "leaky gut" phenotype in a sex-dependent manner and cKO of both $A h R$ and PPAR $\gamma$ resulted in alleviation of pelvic allodynia in female mice. Our findings suggest that dysbiosis associated with AOAH deficiency is mediated by AhR and PPAR $\gamma$ in CRFexpressing cells and reveal novel therapeutic targets for treating pelvic pain. 


\section{MATERIALS AND METHODS}

Animals. Ten to twelve-week-old male and female wild type (WT) C57BL/ 6 mice were purchased from The Jackson Laboratory. Aoah-/- mice (B6.129S6-Aoahtm1Rsm/J) were a gift from Dr. Robert Munford of NIAID and maintained on a 12h:12h light:dark cycle as previously described (2). Conditional knockout mice were generated as previously described in Aguiniga et al. (2)

Pelvic allodynia. Pelvic allodynia was measured by measuring responses to von Frey filament stimulation to the pelvic region, as previously described in Rudick et al. (28). Briefly, pelvic allodynia was measured for WT and AOAH-deficient mice with or without conditional knockout of $A h R$ and/ or PPAR $\gamma$. Mice were placed and allowed to acclimate to the test chamber for $5 \mathrm{~min}$. Starting with the filament that applied the lowest force, five von Frey filaments were applied 10 times to the pelvic region. A behavioral response was measured as painful if the animal jumped, shook the hind paws, or excessively licked the pelvic region.

Gut microbiome analyses. Fecal gut microbiota were analyzed as previously reported in Rahman-Enyart et al. (26). Briefly, DNA was extracted from fecal pellets from male and female mice using the QIAamp DNA Stool Mini Kit (QIAGEN, Hilden, Germany) and homogenized using $0.1 \mathrm{~mm}$ zirconia/silica beads (Biospec, Bartlesville, OK) in 1.4 mL ASL buffer (QIAGEN). Amplicon sequencing of the 16S rRNA V3-V5 hypervariable region was carried out to identify phylotype profiles of microbiota. The V3-V5 hypervariable region was amplified through 30 cycles with primers 357F (CCTACGGGAGGCAGCAG) and 926R (CCGTCAATTCMTTTRAGT). Amplicon pools were quantified on a Qubit fluorimeter, and fragment sizes were determined by Agilent bioanalyzer High Sensitivity DNA LabChip (Agilent Technologies, Wilmington, DE). Amplicons were spiked with a PhiX control library to $20 \%$ and mixtures were then sequenced on an Illumina MiSeq V2 (250nt from each end). Sequence reads were binned at 97\% identity using QIIME and Galaxy in order to define abundance of OTUs and their phylogenic relationships.

Transepithelial electrical resistance. Cecum permeability was measured as transepithelial electrical resistance (TEER) as previously described in Rahman-Enyart et al. (26). 
Ceca were bisected and placed in Ringer's solution at room temperature followed by mounting onto cassettes with $0.126 \mathrm{~cm}^{2}$ aperture. Cassettes were inserted into an Ussing chamber (Physiologic Instruments EM-CSYS-2) with $\mathrm{KCl}$ saturated salt-bridge electrodes. The chambers were then filled with Ringer's solution and bubbled with carbogen $\left(95 \% \quad \mathrm{O}_{2} / 5 \% \quad \mathrm{CO}_{2}\right)$. The chambers were maintained at $37^{\circ} \mathrm{C}$ until resistance was stabilized, about $1 \mathrm{hr}$. Voltage was clamped and current was passed every $3 \mathrm{~s}$ at $30 \mathrm{~s}$ intervals using a VCC MC2 multichannel voltage-current clamp amplifier using Acquire and Analyze software v2.3.4 (Physiologic Instruments).

Statistical analysis. Results were statistically analyzed by implementing either Student's t-test or one-way analysis of variance (ANOVA) followed by Tukey's Multiple comparisons test. All statistics were done using Prism software, version 6 (GraphPad, Inc). Differences were considered statistically significant at $\mathrm{P}<0.05$. 


\section{RESULTS}

\section{Conditional knockout of PPAR $\gamma$ and $A h R$ alleviates pelvic pain in AOAH-deficient mice.}

AOAH-deficient mice exhibit increased pelvic pain behaviors compared to wild-type controls (37). To determine whether the $\mathrm{cKO}$ of $A h r$ or $P P A R \gamma$ in CRF-expressing cells could alter pelvic allodynia in female AOAH-deficient mice, we quantified allodynia in response to von Frey filaments applied to the pelvic region (Fig. 1). Similar to our previous findings (37), we observed that AOAH-deficient mice exhibited a robust pelvic pain phenotype. We observed a significant reduction $(\sim 40 \%)$ in pelvic allodynia in AOAH-deficient mice with a cKO of both $A h R$ and $P P A R \gamma$. The cKO of $A h R$ only did not alleviate the pelvic pain phenotype in AOAH-deficient mice. We observed a slight reduction in pelvic allodynia $(\sim 20 \%)$ in AOAH-deficient mice with a cKO of $P P A R r$, however, these results did not approach significance. These data suggest that both $\mathrm{AhR}$ and PPAR $\gamma$ are required for the pelvic pain phenotype observed in AOAH-deficient mice and the cKO of just one of these genes is not sufficient to alleviate pelvic allodynia.

\section{Conditional knockout of $P P A R \gamma$ and $A h R$ alters fecal microbiota.}

We have previously shown that AOAH-deficient mice exhibit an altered gut microbiome and manipulation of the microbiota alters the pelvic pain phenotype (26). Therefore, we next sought to identify whether the cKO of $A h r$ or $P P A R \gamma$ in AOAH-deficient mice would result in a transformed gut microbiome. We utilized $16 \mathrm{~S}$ rRNA amplicon sequencing data to identify large compositional differences in fecal microbiota between wild type and AOAH-deficient mice with or without cKO of $A h r$ or $\operatorname{PPAR} \gamma$ (Fig. 2). As shown by principal component analysis (PCA, Fig. 2A) and the corresponding heat map (Fig. 2B), AOAH-deficient mice exhibit an altered gut microbiota compared to wild type control, similar to our previous findings (26). Mice with cKO for Ahr or PPAR $\gamma$ show group variance along the PC1 (vertical) axis in bacterial composition of gut flora compared to AOAH-deficient and wild type mice, suggesting an altered but unique microbiome (Fig. 2A). We observed that the gut microbiome of AOAH-deficient mice with cKO for PPAR $\gamma$ was most similar to wild type control, whereas $\mathrm{cKO}$ of $A h R$ was more similar to 
AOAH-deficient microbiota. The cKO of PPAR $\gamma$ in wild type mice also showed an altered microbiome, suggesting a role for $P P A R \gamma$ in regulating bacterial composition of gut flora. Overall, these data identify a role for PPAR $\gamma$ in regulating the gut dysbiosis phenotype in AOAH-deficient mice.

\section{Conditional knockout of PPAR $\gamma$ and $A h R$ alters gut permeability in AOAH-deficient mice.}

Previous studies have linked gut dysbiosis and gut permeability $(9,20,36)$, and we have observed a "leaky gut" phenotype in AOAH-deficient mice (26). To identify whether AhR and PPAR $\gamma$ play a role in gut "leakiness," we assessed cecum barrier function in male and female wild type and AOAH-deficient mice with $\mathrm{cKO}$ of $A h r$ and/or $P P A R \gamma$ by quantifying cecum transepithelial electrical resistance (TEER) ex vivo via an Üssing chamber (Fig. 3A and B, respectively). As we have previously reported (26), cecum TEER was significantly lower in AOAH-deficient mice compared to wild type in both males $\left(27.66 \pm 6.63 \Omega \bullet \mathrm{cm}^{2}\right.$ for wild type and $17.11 \pm 6.11 \Omega \bullet \mathrm{cm}^{2}$ for Aoah ${ }^{-\vdash}$; Fig. 3A) and females $\left(33.58 \pm 4.77 \Omega \bullet \mathrm{cm}^{2}\right.$ for wild type and $19.94 \pm$ $6.66 \Omega \bullet \mathrm{cm}^{2}$ for $A o a h^{--} ;$Fig. 3B). The cKO of PPAR $\gamma$ or both $A h R / P P A R \gamma$ in male AOAH-deficient mice resulted in a significant increase in TEER $\left(36.25 \pm 4.12 \Omega \bullet \mathrm{cm}^{2}\right.$ for PPAR $\gamma \mathrm{cKO}$ and $35.10 \pm$ $4.36 \Omega \bullet \mathrm{cm}^{2}$ for $A h r / P P A R \gamma \mathrm{cKO}$; Fig. 3A), compared to male AOAH-deficient mice. In contrast, in female mice, the cKO of $A h R$ or both $A h R / P P A R \gamma$ resulted in a significant increase in TEER $\left(29.90 \pm 3.79 \Omega \bullet \mathrm{cm}^{2}\right.$ for $A h R \mathrm{cKO}$ and $29.03 \pm 6.31 \Omega \bullet \mathrm{cm}^{2}$ for $A h r / P P A R \gamma \mathrm{cKO}$; Fig. 3B), compared to female AOAH-deficient mice. These data exhibit that both AhR and PPAR $\gamma$ play a role in regulating gut permeability in AOAH-deficient mice, and do so in a sex-dependent manner.

\section{$P P A R Y$ and $A h R$ regulate sex-dependent alterations in AOAH-deficient microbiota.}

Since our observations indicate sex differences in gut permeability regulated by AhR and PPAR $\gamma$ (Fig. 3), we next addressed whether bacterial composition of the gut would be altered in a sex-dependent manner after $\mathrm{cKO}$ of $A h R$ and/or PPAR $\gamma$ in AOAH-deficient mice. Corroborating our data from Fig. 2, 16S rRNA amplicon sequencing indicated that the cKO of 
$A h R$ or $P P A R \gamma$ in female AOAH-deficient mice resulted in a gut microbiome that was distinct from female AOAH-deficient mice (Fig. 4, left column). In addition, we also observed that the cKO of both $A h r / P P A R \gamma$ resulted in an altered fecal microbiota in female AOAH-deficient mice. Female AOAH-deficient mice with cKO for PPAR $\gamma$ or both $A h r / P P A R \gamma$ show a larger group variance along the PC1 (vertical) axis in bacterial composition of gut flora compared to female AOAH-deficient mice and AOAH-deficient mice with cKO for only AhR. These data suggest that both AhR and, to a greater degree, $\operatorname{PAAR} \gamma$ play a role in regulating the gut microbiome in female AOAH-deficient mice.

We next utilized $16 \mathrm{~S}$ rRNA amplicon sequencing in male AOAH-deficient mice to identify whether the conditional knockout of $A h R$ and/or PPAR $\gamma$ would result in altered gut bacterial composition similar to our observations in females. In contrast to female mice, we observed that in male AOAH-deficient mice the cKO of PPAR $\gamma$ or both Ahr / PPAR $\gamma$, but not $A h R$ only, resulted in a gut microbiome that was distinct from AOAH-deficient mice (Fig. 4, right column). These findings suggest a sex difference in the role of AhR and PPAR $\gamma$ in regulating gut bacterial composition, where both $\mathrm{AhR}$ and PPAR $\gamma$ play a role in female gut flora but only PPAR $\gamma$ play a role in male flora.

To determine the specificity of AhR and PPAR $\gamma$ modulation of AOAH-deficient gut microbiota, we examined the relative abundance of bacterial phyla (Fig. 5). As expected, the majority of bacteria identified in all mouse groups belonged to Bacteroidetes and Firmicutes phyla. Similar to our previous findings (26), we did not observe differences in mean bacterial abundance belonging to Bacteroidetes and Firmicutes phyla in wild type and AOAH-deficient conditions, but did observe differences within less abundant phyla (Fig. 5). In contrast, we observed that the cKO of $P P A R \gamma$ or both $A h r / P P A R \gamma$ resulted in a decrease in Firmicutes phyla when compared to AOAH-deficient and wild type mice (Fig. 5 C-F). We observed an increase in abundance in Bacteroidetes phyla in male AOAH-deficient mice with cKO of $A h r / P P A R \gamma$, but not in females (Fig. 5E and F). In addition, the $\mathrm{cKO}$ of $A h R$ resulted in greater abundance of the less common Cyanobacteria and Proteobacteria phyla in both males and females when compared 
to AOAH-deficient and wild type mice (Fig. 5A and B). We observed sex differences in the abundance of bacteria belonging to Actinobacteria phyla, where male AOAH-deficient mice with cKO for Ahr and/or PPAR $\gamma$ exhibited greater abundance compared to AOAH-deficient and wild type mice, which was not observed in females (Fig. 5B, D, and F compared to Fig. 5A, C, and E).

In addition, we analyzed relative abundance of Firmicutes and Bacteroidetes phyla in response to cKO of $A h R, P P A R \gamma$, or both $A h r / P P A R \gamma$. Relative abundance of bacteria belonging to Firmicutes phyla was greater in AOAH-deficient mice compared to WT mice; whereas, bacteria belonging to Bacteroidetes phyla was more abundant in WT compared to AOAH-deficient mice (Fig. 6). We observed that the cKO of $A h R, P P A R \gamma$, or both $A h r / P P A R \gamma$ altered relative abundance of both Firmicutes and Bacteroidetes phyla to abundance that more closely resembled wild type. Of note, in females, we observed a greater change in mice with a conditional knockout for PPAR $\gamma$ compared to $A h R$ (Fig. 6E and F compared to Fig.6A and B). Overall, these data show that both $\mathrm{AhR}$ and PPAR $\gamma$ play important roles in regulating the gut microbiome in AOAH-deficient mice, and their effects are sex-dependent. 


\section{DISCUSSION}

AOAH deficiency recapitulates several aspects of IC/BPS and we have previously identified Aoah as a genetic modulator of gut microbiota composition (1, 2, 26, 37). Here we show that AhR and PPAR $\gamma$ in CRF-expressing cells can modulate gut dysbiosis mediated by AOAH deficiency. CRF is best known for its role in the stress response through regulating the HPA axis (24). In addition, CRF also plays a crucial role in pain modulation, gut function, and composition of the gut microbiome $(11,16,33,34)$. We have previously shown increased Crf expression in the paraventricular nucleus of AOAH-deficient mice as well as elevated serum corticosterone (2). In addition, cKO of $A h r$ and $P P A R \gamma$, two lipid-responsive transcription regulators of Crf, can alleviate voiding dysfunction and depressive behaviors in AOAH-deficient mice $(1,2)$. Increased activation of mast cell CRFR1 receptors via elevated CRF in the enteric epithelium has been observed to increase epithelial permeability (27), a phenotype often observed in gut dysbiosis (13). Consistent with these data, we observed increased leakiness in ceca of AOAH-deficient mice that improved upon the $\mathrm{cKO}$ of $A h R$ or $P P A R \gamma$ (Fig. 3), suggesting a role for CRF in gut epithelial permeability in AOAH-deficient mice.

Although we observed changes in pelvic allodynia, gut microbiome composition, and TEER upon manipulation of Crf expression in AOAH-deficient mice, we identified that the effects of $A h R, P P A R \gamma$, or both $A h R / P P A R \gamma$ was dependent on phenotype and sex. The cKO of PPAR $\gamma$ had a greater role in improving TEER and altering the gut microbiome in males compared to the cKO of $A h R$ (Figs. 3 and 4). In female AOAH-deficient mice, both AhR and PPAR $\gamma$ play a role in altering the gut microbiota (Fig. 4), but AhR is the key driver in modulating gut permeability (Fig. 3). Additionally, the $\mathrm{cKO}$ of both $A h R / P P A R \gamma$ was required to alleviate the pelvic pain phenotype in females (Fig. 1). Our findings suggest that both AhR and PPAR $\gamma$ are regulators of gut function but possess unique roles that are sex-dependent. Sex-specific differences in gut microbiota have been observed in both mice and humans (15). Unsurprisingly, sex hormones such as testosterone and estrogen are directly linked to altered gut microbiota. In addition, metabolism, body mass 
index, and colonic transit time differ amongst sex and can also alter gut flora (15). Whether AhR and PPAR $\gamma$-dependent transcription of Crf modulates these sex-dependent biological functions in AOAH-deficient mice will require further investigation.

Manipulation of PPAR $\gamma$ and AhR signaling has been associated with microbiota alterations and gut permeability $(5,23)$. Depletion of butyrate-producing microbes reduces PPAR $\gamma$ signaling in epithelial cells of the colon; whereas, induction of microbiota-dependent $\operatorname{PPAR} \gamma$ signaling drives homeostasis by preventing the dysbiotic expansion of Enterobacteriacae (phylum: Proteobacteria) (5). Our findings did not indicate altered Proteobacteria in AOAHdeficient mice with $\mathrm{cKO}$ for $P P A R \gamma$, however, we did see increase abundance in Proteobacteria in $A h R$ cKOs (Fig. 5), suggesting that AhR is the driving factor for the presence of Proteobacteria in AOAH-deficient mice.

Furthermore, we observed altered mean abundance in bacteria belonging to Firmicutes phylum in AOAH-deficient mice with a $\mathrm{cKO}$ of $P P A R \gamma$ or both $A h r / P P A R \gamma$ (Fig. 5). A previous study demonstrated that mice fed a high-fat diet showed drastic changes in microbiota composition, including an increase in abundance of Firmicutes as well as dysregulation of the PPAR $\gamma$ pathway (35). In addition, oral administration of water-insoluble polysaccharides to treat a mouse model of alcoholic hepatic steatosis demonstrated both an increase in abundance of Firmicutes and PPAR $\gamma$ signaling (32), suggesting a correlation between expression of Firmicutes and PPAR $\gamma$ signaling. In conjunction to these findings, we observed increased relative abundance of bacteria belonging to Firmicutes phylum in AOAH-deficient mice, which was lower in AOAHdeficient mice with a cKO of $P P A R \gamma$ (Fig. 6). Interestingly, acute pain perception has also been associated with altered gut composition, including increase in Firmicutes abundance (29), suggesting a role for bacteria belonging to Firmicutes phylum in pain. Indeed, our findings showed that the cKO of both $A h r / P P A R \gamma$ resulted in a significant decrease in pelvic allodynia in AOAH-deficient mice (Fig. 1).

Metabolites derived from the metabolism of tryptophan have been shown to signal through AhR, and aberrant production of tryptophan-based AhR ligands have been observed in 
the pathogenesis of inflammatory bowel disease $(17,38)$. Indeed, we observed that both AhR and PPAR $\gamma$ can alter gut microbiota composition (Figs. 2 and Figs. 4-6). Additionally, we have previously identified the tryptophan metabolite xanthurenic acid as a gut metabolite overexpressed in AOAH-deficient cecal stool (26). Therefore, increased AhR signaling in AOAHdeficient mice through ligands derived from tryptophan metabolism may be driving phenotypes associated with AOAH deficiency. Our current studies did not identify possible alterations in gut metabolites as a result of $\mathrm{cKO}$ of $A h R$ and $P P A R \gamma$ and would be required to assess whether tryptophan metabolism or other gut metabolites may play a role in AhR and PPAR $\gamma$-mediated gut function and epithelial permeability.

Growing evidence has linked dysbiosis of the gut microbiota with the pathogenesis of several diseases (8). Patients with IC/BPS have altered gut microbiota, a phenotype that AOAHdeficient mice also share $(4,26)$. As the gut microbiome is involved in bidirectional communication with the CNS, unsurprisingly, several neurological disorders such as depression or neuropathic pain are also affected by an altered gut microbiota $(6,11)$. Studies from our lab have shown that manipulating the gut microbiome in AOAH-deficient mice improves phenotypes observed in IC/BPS, including pelvic pain and depressive behaviors (26). Here we showed that AOAH-deficient gut dysbiosis and pelvic pain can be manipulated through Crf transcription regulators AhR and PPAR $\gamma$. These findings are central in our understanding of how gut dysbiosis may be modulating the pelvic pain phenotype in IC/BPS. Both AOAH-deficient mice and IC/BPS exhibit HPA dysregulation (2, 19), suggesting a role for CRF in symptomatology. Therefore, future patient studies addressing the correlation of HPA dysregulation and gut dysbiosis will be an interesting avenue for clinical advances in treating IC / BPS.

In summary, the data presented here show that the gut dysbiosis and compromised gut epithelia exhibited by AOAH-deficient mice is modulated by the transcription regulators of $\mathrm{Crf}$, $\mathrm{AhR}$ and PPAR $\gamma$. The combined function of AhR and PPAR $\gamma$ also play a role in the pelvic pain phenotype observed in AOAH-deficient mice. These findings demonstrate that the gut 
bioRxiv preprint doi: https://doi.org/10.1101/2021.03.04.433950; this version posted March 5, 2021. The copyright holder for this preprint (which was not certified by peer review) is the author/funder. All rights reserved. No reuse allowed without permission.

microbiome and CRF signaling pathways are promising therapeutic targets for the development of IC/BPS treatments. 


\section{ETHICS APPROVAL AND CONSENT TO PARTICIPATE}

All animals were maintained at the Center for Comparative Medicine at Northwestern University and utilized for experimentation under Northwestern IACUC approved protocols.

\section{COMPETING INTERESTS}

The authors declare that they have no competing interests.

\section{FUNDING}

This work was supported by NIH/NIDDK award R01 DK103769 (B.A.W., A.J.S., and D.J.K) and by NIH/NIDDK T32 DK062716 postdoctoral fellowship to Dr. Rahman-Enyart.

\section{AUTHOR CONTRIBUTIONS}

L.M.A., W.Y., A.J.S., and D.J.K conceived and designed research; L.M.A., W.Y., R.E.Y. B.W., M.W., L.A., M.B., and C.B. performed experiments; A.R.-E., W.Y., and R.E.Y. analyzed data; A.R.-E., A.J.S., and D.J.K. interpreted results of experiments; A.R.-E., R.E.Y., and D.J.K. prepared figures; A.R.-E. and D.J.K. drafted manuscript; A.R.-E., A.J.S., and D.J.K. edited and revised manuscript; A.R.-E., L.M.A., W.Y., R.E.Y. B.W., M.W., L.A., M.B., C.B., A.J.S., and D.J.K. approved final version of manuscript.

\section{ACKNOWLEDGEMENTS}

We thank Dr. Robert Munford for generously providing AOAH-deficient mice and for many helpful discussions. 


\section{REFERENCES}

1. Aguiniga LM, Searl TJ, Rahman-Enyart A, Yaggie RE, Yang W, Schaeffer AJ, Klumpp DJ. Acyloxyacyl hydrolase regulates voiding activity. Am J Physiol Renal Physiol 318: F1006-F1016, 2020.

2. Aguiniga LM, Yang W, Yaggie RE, Schaeffer AJ, Klumpp DJ, MAPP Research Network Study Group. Acyloxyacyl hydrolase modulates depressive-like behaviors through aryl hydrocarbon receptor. Am J Physiol Regul Integr Comp Physiol 317: R289-R300, 2019.

3. Akiyama Y, Homma Y, Maeda D. Pathology and terminology of interstitial cystitis/bladder pain syndrome: A review. Histol Histopathol 34: 25-32, 2019.

4. Braundmeier-Fleming A, Russell NT, Yang W, Nas MY, Yaggie RE, Berry M, Bachrach L, Flury SC, Marko DS, Bushell CB, Welge ME, White BA, Schaeffer AJ, Klumpp DJ. Stool-based biomarkers of interstitial cystitis / bladder pain syndrome. Sci Rep 6: 26083, 2016.

5. Byndloss MX, Olsan EE, Rivera-Chávez F, Tiffany CR, Cevallos SA, Lokken KL, Torres TP, Byndloss AJ, Faber F, Gao Y, Litvak Y, Lopez CA, Xu G, Napoli E, Giulivi C, Tsolis RM, Revzin A, Lebrilla CB, Bäumler AJ. Microbiota-activated PPAR- $\gamma$ signaling inhibits dysbiotic Enterobacteriaceae expansion. Science 357: 570-575, 2017.

6. Capuco A, Urits I, Hasoon J, Chun R, Gerald B, Wang JK, Kassem H, Ngo AL, AbdElsayed A, Simopoulos T, Kaye AD, Viswanath O. Current perspectives on gut microbiome dysbiosis and depression. Adv Ther 37: 1328-1346, 2020.

7. Defaye M, Gervason S, Altier C, Berthon J-Y, Ardid D, Filaire E, Carvalho FA. Microbiota: a novel regulator of pain. J Neural Transm 127: 445-465, 2020.

8. DeGruttola AK, Low D, Mizoguchi A, Mizoguchi E. Current understanding of dysbiosis in disease in human and animal models. Inflamm Bowel Dis 22: 1137-1150, 2016.

9. Dupont HL, Jiang Z-D, Dupont AW, Utay NS. The intestinal microbiome in human 
health and disease. Trans Am Clin Climatol Assoc 131: 178-197, 2020.

10. Erwin AL, Munford RS. Deacylation of structurally diverse lipopolysaccharides by human acyloxyacyl hydrolase. J Biol Chem 265: 16444-16449, 1990.

11. Guo R, Chen L-H, Xing C, Liu T. Pain regulation by gut microbiota: molecular mechanisms and therapeutic potential. Br J Anaesth 123: 637-654, 2019.

12. Hagen FS, Grant FJ, Kuijper JL, Slaughter CA, Moomaw CR, Orth K, O'Hara PJ, Munford RS. Expression and characterization of recombinant human acyloxyacyl hydrolase, a leukocyte enzyme that deacylates bacterial lipopolysaccharides. Biochemistry 30: 8415-8423, 1991.

13. Kelly JR, Kennedy PJ, Cryan JF, Dinan TG, Clarke G, Hyland NP. Breaking down the barriers: the gut microbiome, intestinal permeability and stress-related psychiatric disorders. Front Cell Neurosci 9: 392, 2015.

14. Kim H-J. Update on the pathology and diagnosis of interstitial cystitis/bladder pain syndrome: A review. Int Neurourol J 20: 13-17, 2016.

15. Kim YS, Unno T, Kim BY, Park MS. Sex differences in gut microbiota. World J Mens Health 38: 48-60, 2020.

16. Lach G, Schellekens H, Dinan TG, Cryan JF. Anxiety, depression, and the microbiome: A role for gut peptides. Neurotherapeutics 15: 36-59, 2018.

17. Lamas B, Richard ML, Leducq V, Pham H-P, Michel M-L, Da Costa G, Bridonneau C, Jegou S, Hoffmann TW, Natividad JM, Brot L, Taleb S, Couturier-Maillard A, NionLarmurier I, Merabtene F, Seksik P, Bourrier A, Cosnes J, Ryffel B, Beaugerie L, Launay J-M, Langella P, Xavier RJ, Sokol H. CARD9 impacts colitis by altering gut microbiota metabolism of tryptophan into aryl hydrocarbon receptor ligands. Nat Med 22: 598-605, 2016.

18. Lu M, Varley AW, Ohta S, Hardwick J, Munford RS. Host inactivation of bacterial lipopolysaccharide prevents prolonged tolerance following gram-negative bacterial infection. Cell Host Microbe 4: 293-302, 2008. 
19. Lutgendorf SK, Kreder KJ, Rothrock NE, Hoffman A, Kirschbaum C, Sternberg EM, Zimmerman MB, Ratliff TL. Diurnal cortisol variations and symptoms in patients with interstitial cystitis. J Urol 167: 1338-1343, 2002.

20. Lynch SV, Pedersen O. The human intestinal microbiome in health and disease. N Engl J Med 375: 2369-2379, 2016.

21. Mullins C, Bavendam T, Kirkali Z, Kusek JW. Novel research approaches for interstitial cystitis/bladder pain syndrome: thinking beyond the bladder. Transl Androl Urol 4: 524533, 2015.

22. Munford RS, Hunter JP. Acyloxyacyl hydrolase, a leukocyte enzyme that deacylates bacterial lipopolysaccharides, has phospholipase, lysophospholipase, diacylglycerollipase, and acyltransferase activities in vitro. J Biol Chem 267: 10116-10121, 1992.

23. Natividad JM, Agus A, Planchais J, Lamas B, Jarry AC, Martin R, Michel M-L, ChongNguyen C, Roussel R, Straube M, Jegou S, McQuitty C, Le Gall M, da Costa G, Lecornet E, Michaudel C, Modoux M, Glodt J, Bridonneau C, Sovran B, Dupraz L, Bado A, Richard ML, Langella P, Hansel B, Launay J-M, Xavier RJ, Duboc H, Sokol H. Impaired aryl hydrocarbon receptor ligand production by the gut microbiota is a key factor in metabolic syndrome. Cell Metab 28: 737-749.e4, 2018.

24. Orth DN, Jackson RV, DeCherney GS, DeBold CR, Alexander AN, Island DP, Rivier J, Rivier C, Spiess J, Vale W. Effect of synthetic ovine corticotropin-releasing factor. Dose response of plasma adrenocorticotropin and cortisol. J Clin Invest 71: 587-595, 1983.

25. Pusceddu MM, Gareau MG. Visceral pain: gut microbiota, a new hope? J Biomed Sci 25: 73, 2018.

26. Rahman-Enyart A, Yang W, Yaggie RE, White B, Welge M, Auvil L, Berry M, Bushell C, Rosen JM, Rudick CN, Schaeffer AJ, Klumpp DJ. Acyloxyacyl Hydrolase is a Host Determinant of Gut Microbiome-Mediated Pelvic Pain [Online]. BioRxiv. https: / / www.biorxiv.org/ content/10.1101/2021.01.27.428290v1.abstract [5 Feb. 2021].

27. Rodiño-Janeiro BK, Alonso-Cotoner C, Pigrau M, Lobo B, Vicario M, Santos J. Role of 
Corticotropin-releasing Factor in Gastrointestinal Permeability. J Neurogastroenterol Motil 21: 33-50, 2015.

28. Rudick CN, Chen MC, Mongiu AK, Klumpp DJ. Organ cross talk modulates pelvic pain. Am J Physiol Regul Integr Comp Physiol 293: R1191-8, 2007.

29. Shiro Y, Arai Y-C, Ikemoto T, Ueda W, Ushida T. Correlation between gut microbiome composition and acute pain perception in young healthy male subjects. Pain Med. ( December 1, 2020). doi: $10.1093 / \mathrm{pm} / \mathrm{pnaa} 401$.

30. Shreiner AB, Kao JY, Young VB. The gut microbiome in health and in disease. Curr Opin Gastroenterol 31: 69-75, 2015.

31. Staab JF, Ginkel DL, Rosenberg GB, Munford RS. A saposin-like domain influences the intracellular localization, stability, and catalytic activity of human acyloxyacyl hydrolase. J Biol Chem 269: 23736-23742, 1994.

32. Sun S, Wang K, Sun L, Cheng B, Qiao S, Dai H, Shi W, Ma J, Liu H. Therapeutic manipulation of gut microbiota by polysaccharides of Wolfiporia cocos reveals the contribution of the gut fungi-induced PGE2 to alcoholic hepatic steatosis. Gut Microbes 12: 1830693, 2020.

33. Tache $\mathbf{Y}$, Larauche M, Yuan P-Q, Million M. Brain and gut CRF signaling: biological actions and role in the gastrointestinal tract. Curr Mol Pharmacol 11: 51-71, 2018.

34. Taché Y, Perdue MH. Role of peripheral CRF signalling pathways in stress-related alterations of gut motility and mucosal function. Neurogastroenterol Motil 16 Suppl 1: 137142, 2004.

35. Tomas J, Mulet C, Saffarian A, Cavin J-B, Ducroc R, Regnault B, Kun Tan C, Duszka K, Burcelin R, Wahli W, Sansonetti PJ, Pédron T. High-fat diet modifies the PPAR- $\gamma$ pathway leading to disruption of microbial and physiological ecosystem in murine small intestine. Proc Natl Acad Sci USA 113: E5934-E5943, 2016.

36. Viganò D, Zara F, Usai P. Irritable bowel syndrome and endometriosis: New insights for old diseases. Dig Liver Dis 50: 213-219, 2018. 
37. Yang W, Yaggie RE, Jiang MC, Rudick CN, Done J, Heckman CJ, Rosen JM, Schaeffer AJ, Klumpp DJ. Acyloxyacyl hydrolase modulates pelvic pain severity. Am J Physiol Regul Integr Comp Physiol 314: R353-R365, 2018.

38. Zelante T, Iannitti RG, Cunha C, De Luca A, Giovannini G, Pieraccini G, Zecchi R, D’Angelo C, Massi-Benedetti C, Fallarino F, Carvalho A, Puccetti P, Romani L. Tryptophan catabolites from microbiota engage aryl hydrocarbon receptor and balance mucosal reactivity via interleukin-22. Immunity 39: 372-385, 2013. 


\section{FIGURE LEGENDS}

Figure 1. Conditional knockout of $A h R / P P A R \gamma$ in female AOAH-deficient mice alleviates pelvic allodynia. AOAH-deficient mice exhibited increased pelvic allodynia compared to AOAHdeficient mice with cKO of $A h R / P P A R \gamma$ in CRF-expressing cells, as shown through response to von Frey filaments stimulating the pelvic region $\left(\mathrm{n}=5-12\right.$ mice; ${ }^{*} \mathrm{P}<0.05$, ${ }^{* *} \mathrm{P}<0.01$, Student's t test, two tailed). Data represented as average response $(\%) \pm$ SEM.

Figure 2. Conditional knockout of $A h R$ or $P P A R \gamma$ in female AOAH-deficient mice alters gut microbiome composition. A. PCA plot of $16 \mathrm{~S}$ rRNA analyses of fecal stool in female wild-type and AOAH-deficient mice and mice with a cKO of $A h R$ or PPAR $\gamma$ in CRF-expressing cells. Dots represent individual mice; AOAH-deficient mice shown in blue, wild-type mice shown in green, AOAH-deficient mice with cKO of Ahr shown in yellow, AOAH-deficient mice with cKO of $P P A R \gamma$ shown in purple, WT mice with $\mathrm{cKO}$ of $P P A R \gamma$ shown in red $(\mathrm{n}=3-6$ mice). B. Heat map of $16 \mathrm{~S}$ rRNA analyses of fecal stool from AOAH-deficient (blue), wild-type (red), AOAHdeficient/cKO of $A h r$ (green), AOAH-deficient/cKO of $P P A R \gamma$ (purple), and WT/cKO of PPAR $\gamma$ (orange) female mice ( $\mathrm{n}=3-6$ mice)

Figure 3. Conditional knockout of $A h R$ or $P P A R \gamma$ in AOAH-deficient mice alters TEER. Ceca of male (A) and female (B) mice were measured for transepithelial electrical resistance (TEER). Both male and female AOAH-deficient mice exhibited lower TEER compared to WT. A. The cKO of PPAR $\gamma$ or $A h r / P P A R \gamma$ in male AOAH-deficient mice showed improved TEER compared to AOAH-deficient mice. B. The cKO of $A h r$ or $A h r / P P A R \gamma$ in female AOAH-deficient mice showed improved TEER compared to AOAH-deficient mice. ( $\mathrm{n}=5-9$ mice; ${ }^{*} \mathrm{P}<0.05,{ }^{* *} \mathrm{P}<0.01$, ${ }^{* * *} \mathrm{P}<0.001$, One-Way ANOVA followed by post-hoc Tukey HSD). Data represented as individual values (dots) and average (represented by horizontal line) \pm SEM 
Figure 4. Sex differences in microbiome composition in AOAH-deficient mice with conditional knockout of $A h R$ or $P P A R \gamma$. PCA plots of $16 \mathrm{~S}$ rRNA analyses of fecal stool in female (left column) or male (right column) AOAH-deficient mice with a cKO of AhR (top), PPAR (middle), or AhR / PPAR $\gamma$ (bottom). Dots represent individual mice; Aoah ${ }^{-/-}$Ahr control shown in blue and cKO shown in orange, Aoaht-- PPAR $\gamma$ control shown in yellow and cKO shown in purple, Aoaht-$A h r / P P A R \gamma$ control shown in red and cKO shown in green ( $\mathrm{n}=5$ mice for all conditions)

Figure 5. Quantification of mean abundance of bacterial phyla in female (A, C, and E) and male (B, D, and F) wild-type, AOAH-deficient, and cKO mice using $16 \mathrm{~S}$ rRNA sequencing ( $\mathrm{n}=5$ mice for all conditions)

Figure 6. Relative abundance of Bacteroidetes and Firmicutes phyla in AOAH-deficient stool with conditional knockout of $A h R$ and/or PPAR $\gamma$. The effects on cKO of $A h R$ (A-D), PPAR $\gamma(\mathrm{E}-$ $\mathbf{H})$, or $A h R / P P A R \gamma(\mathbf{I}-\mathbf{L})$ on relative abundance of bacteria belonging to Bacteroidetes and Firmicutes phyla was measured in WT and AOAH-deficient female (A, B, E, F, I, and J) and male (C, D, G, H, K and L) mice using $16 \mathrm{~S}$ rRNA sequencing ( $\mathrm{n}=5$ for all conditions) 
Figure 1 Rahman-Enyart et al

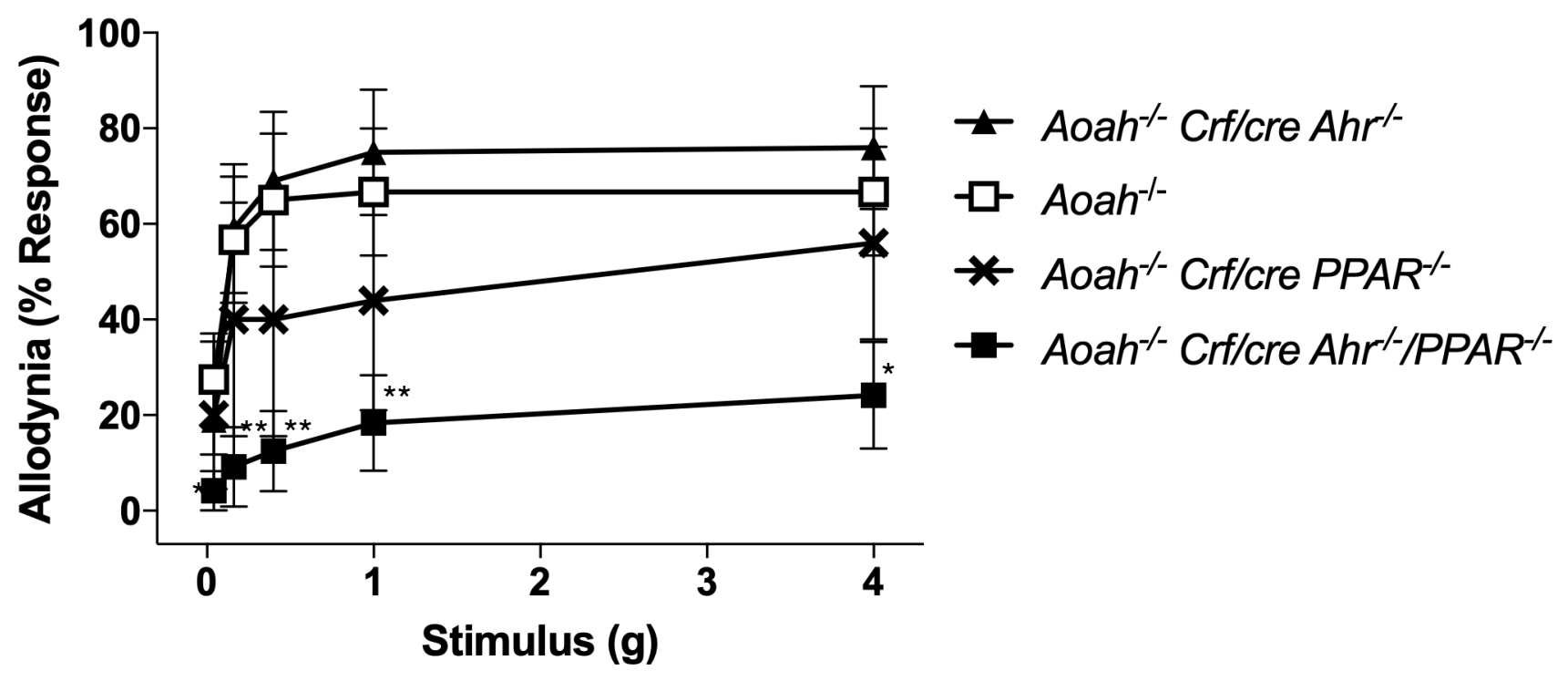


Figure 2 Rahman-Enyart et al

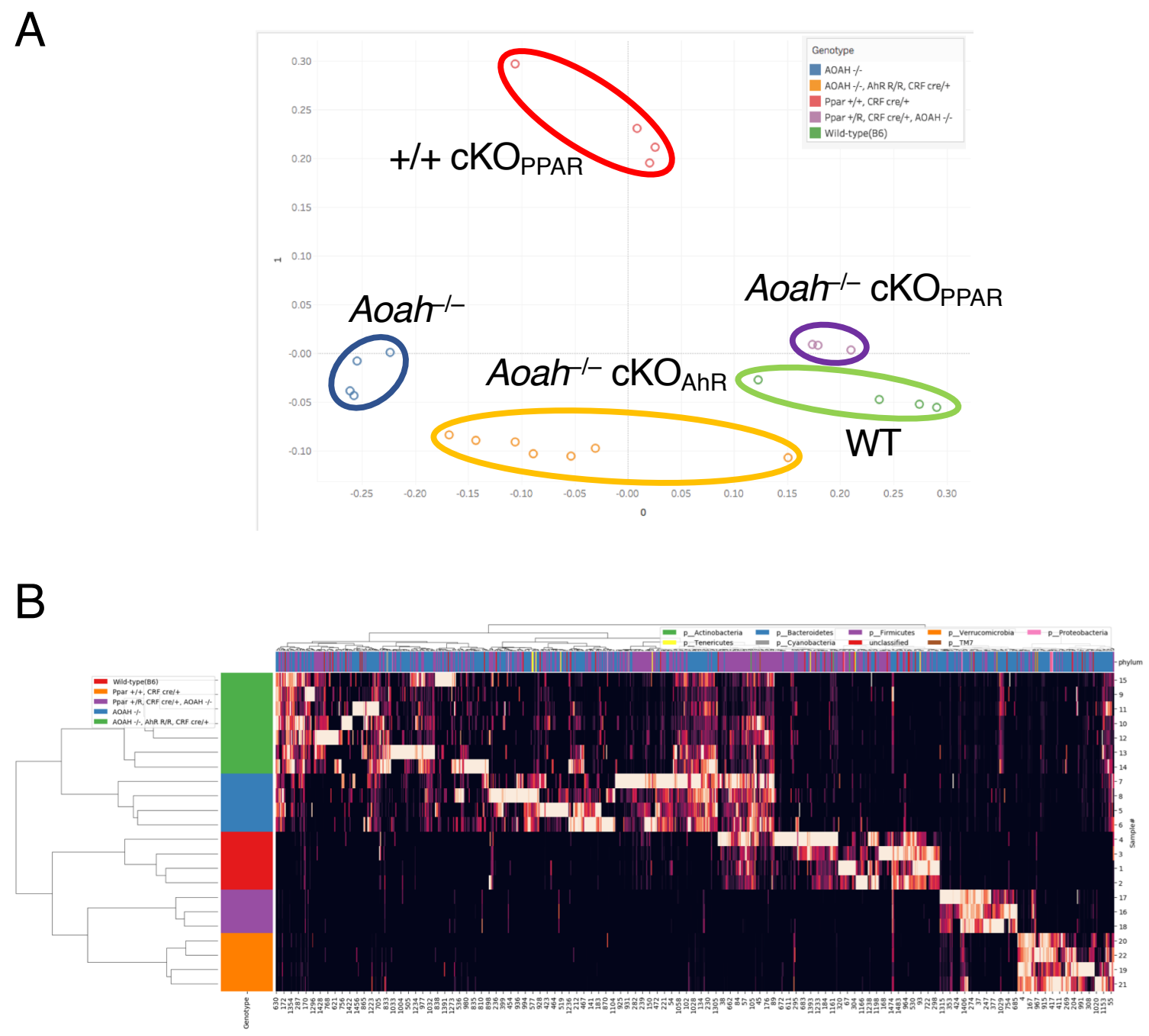


Figure 3 Rahman-Enyart et al

A

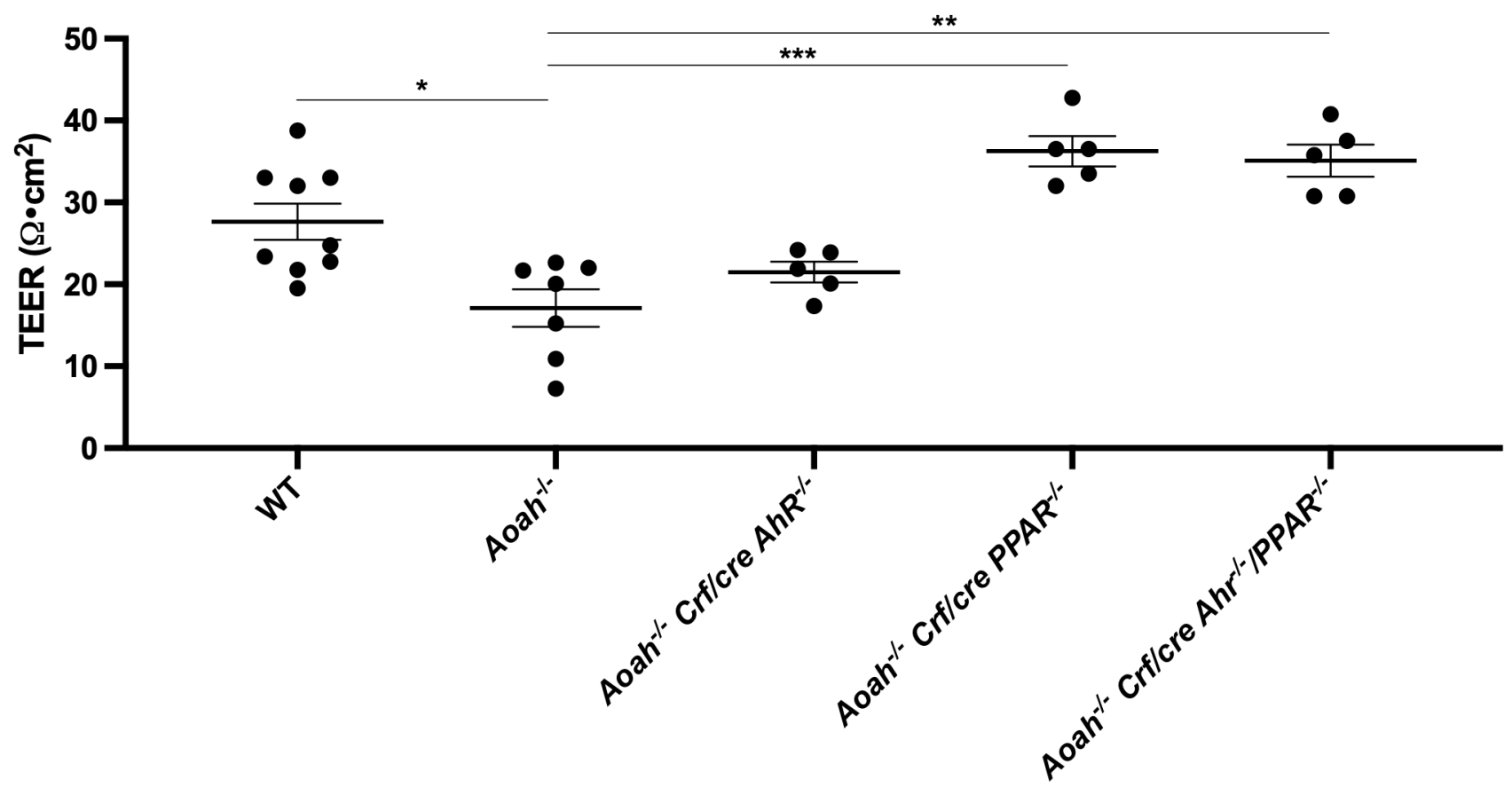

B

Female Cecum

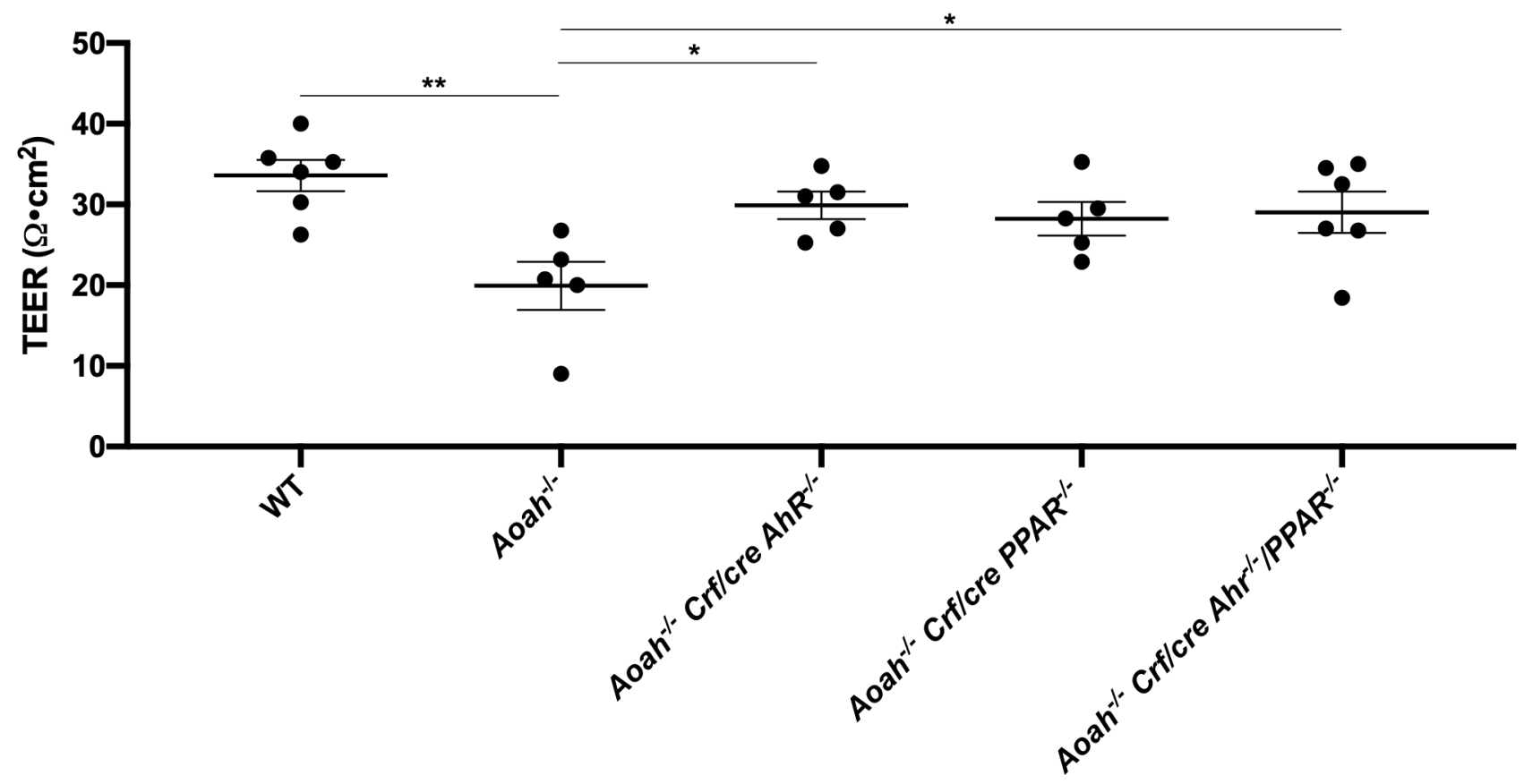


Figure 4. Rahman-Enyart et al

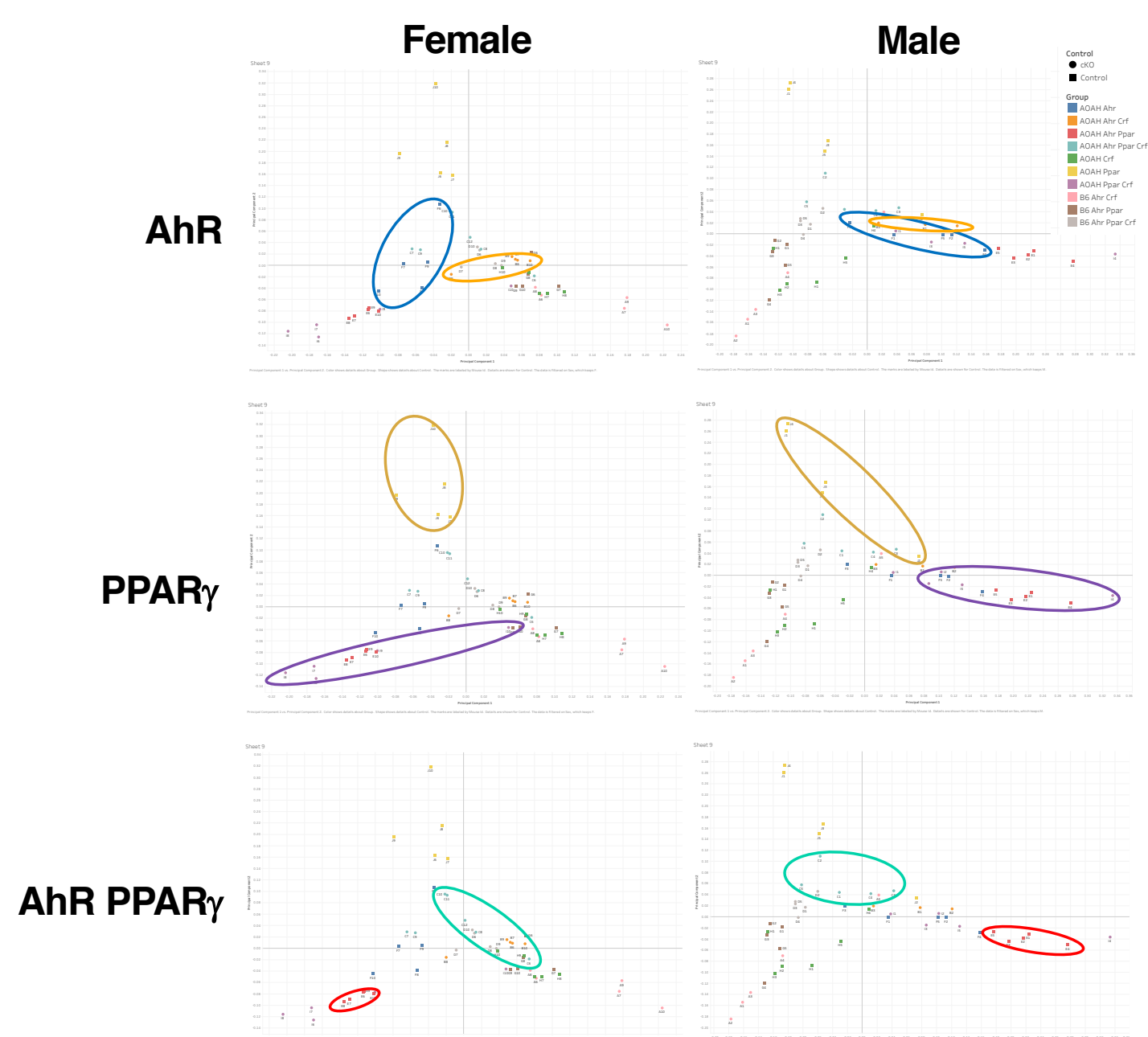


Figure 5. Rahman-Enyart et al

Female
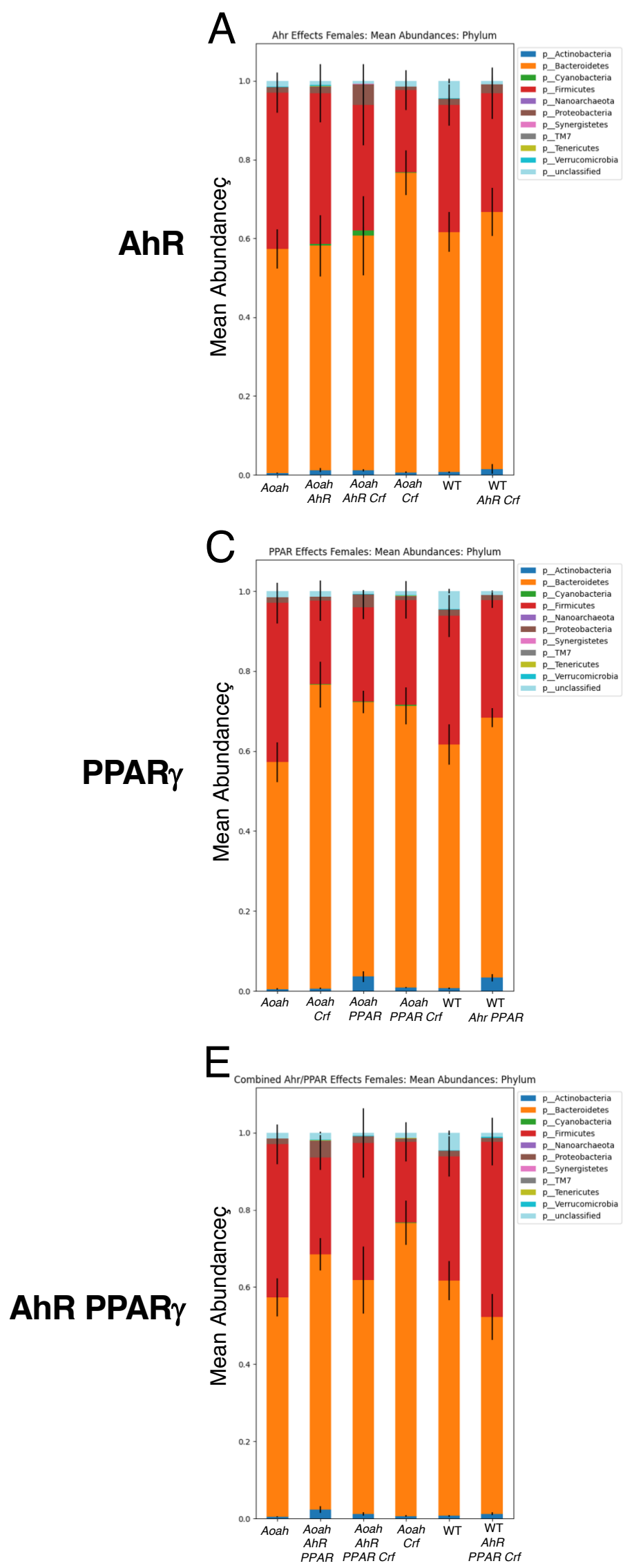

Male
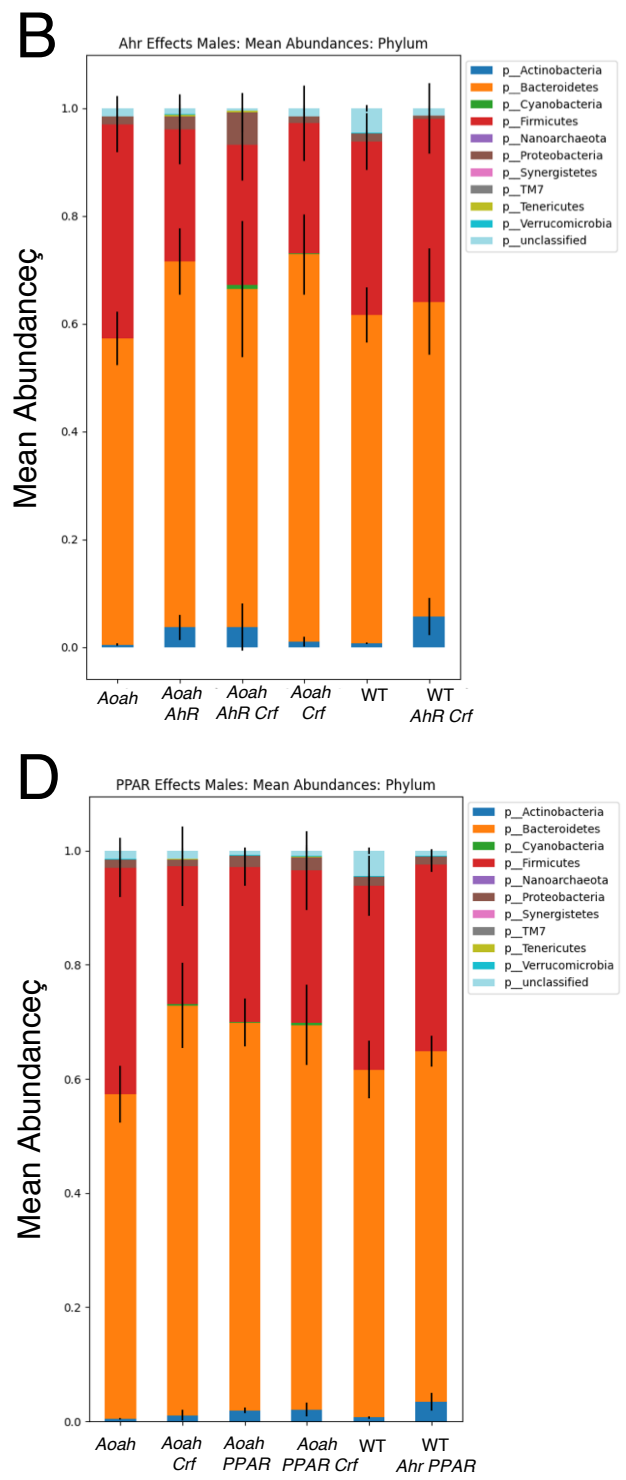

F

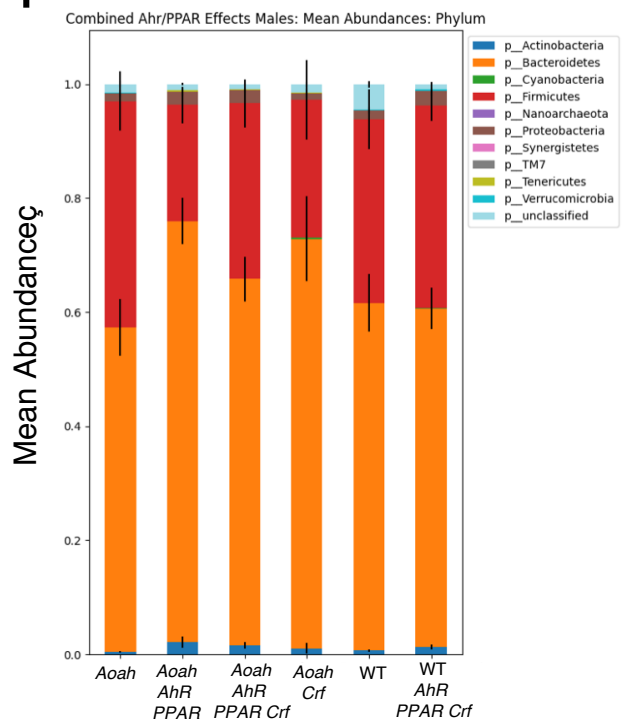


Figure 6. Rahman-Enyart et al

\section{AhR}

A

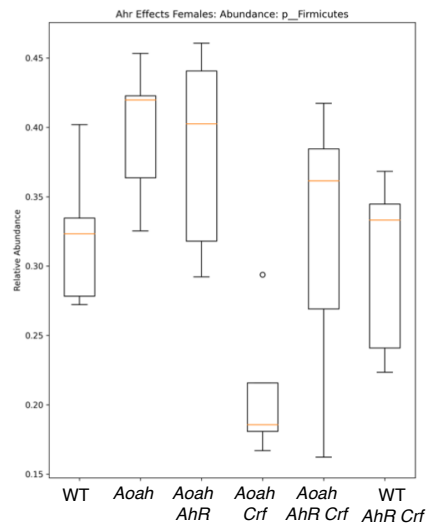

PPAR $\gamma$
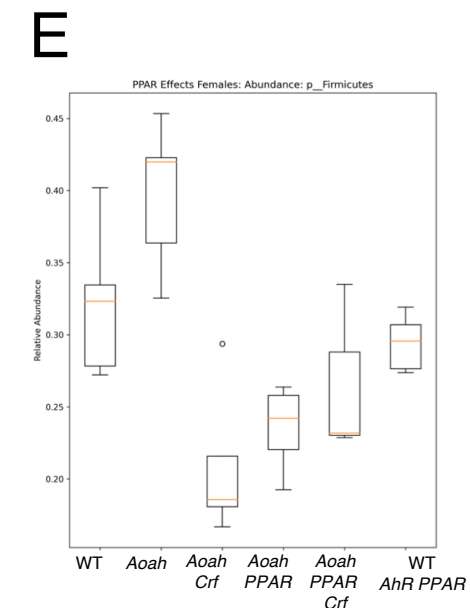

\section{AhR PPAR $\gamma$}

I

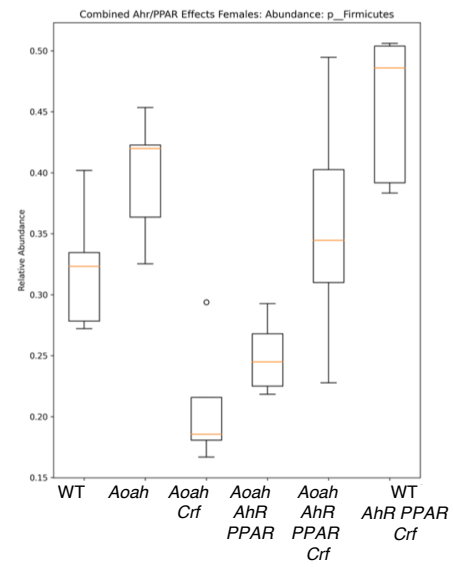

B

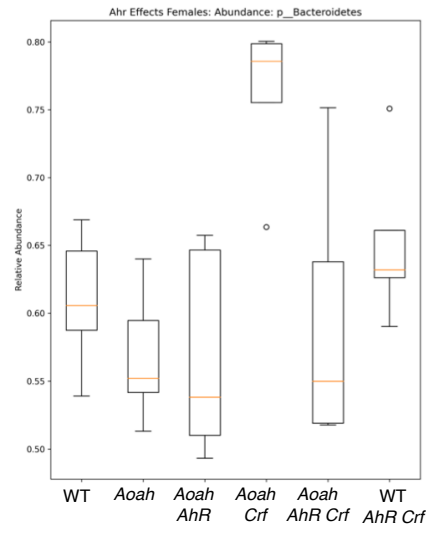

C

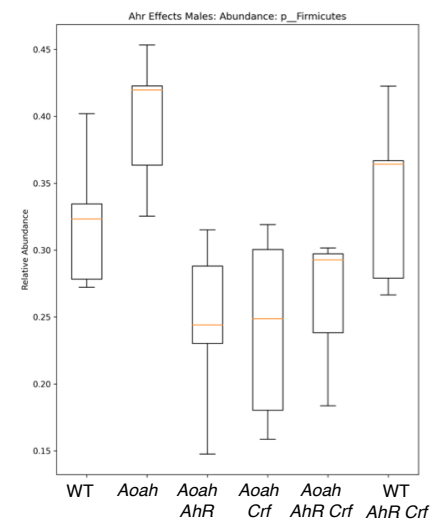

D
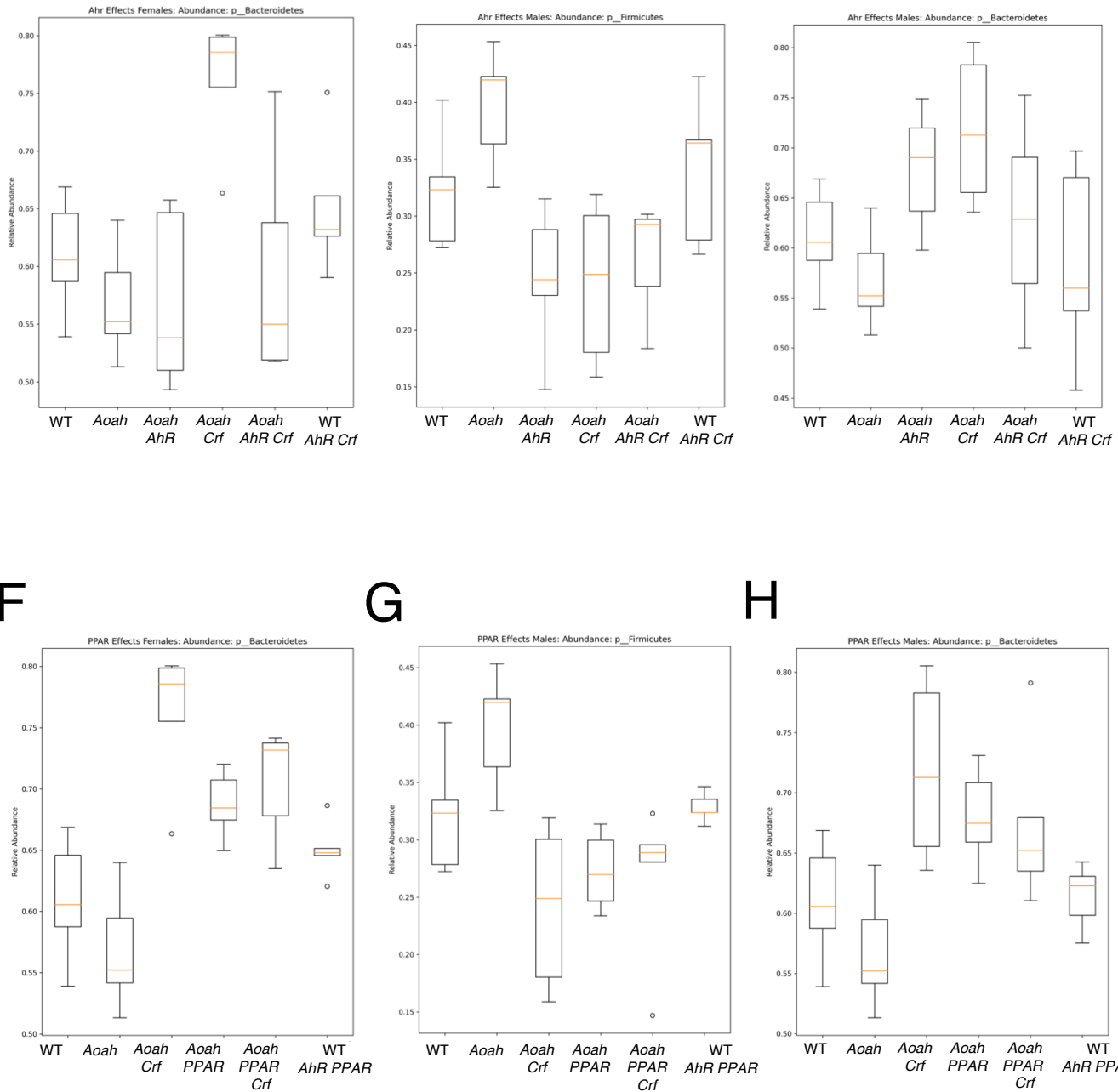

G $\quad H$

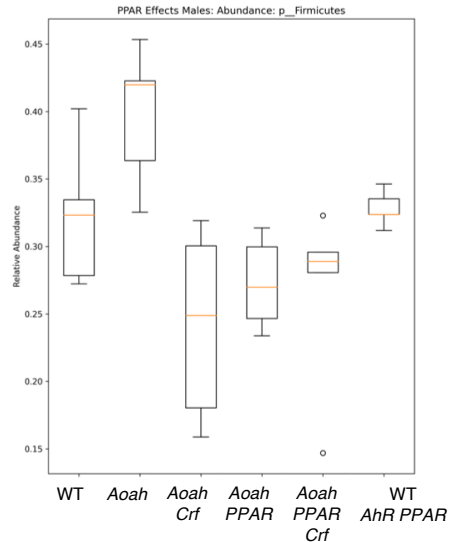

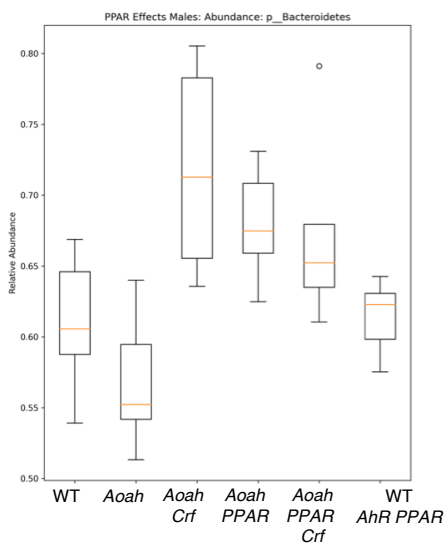
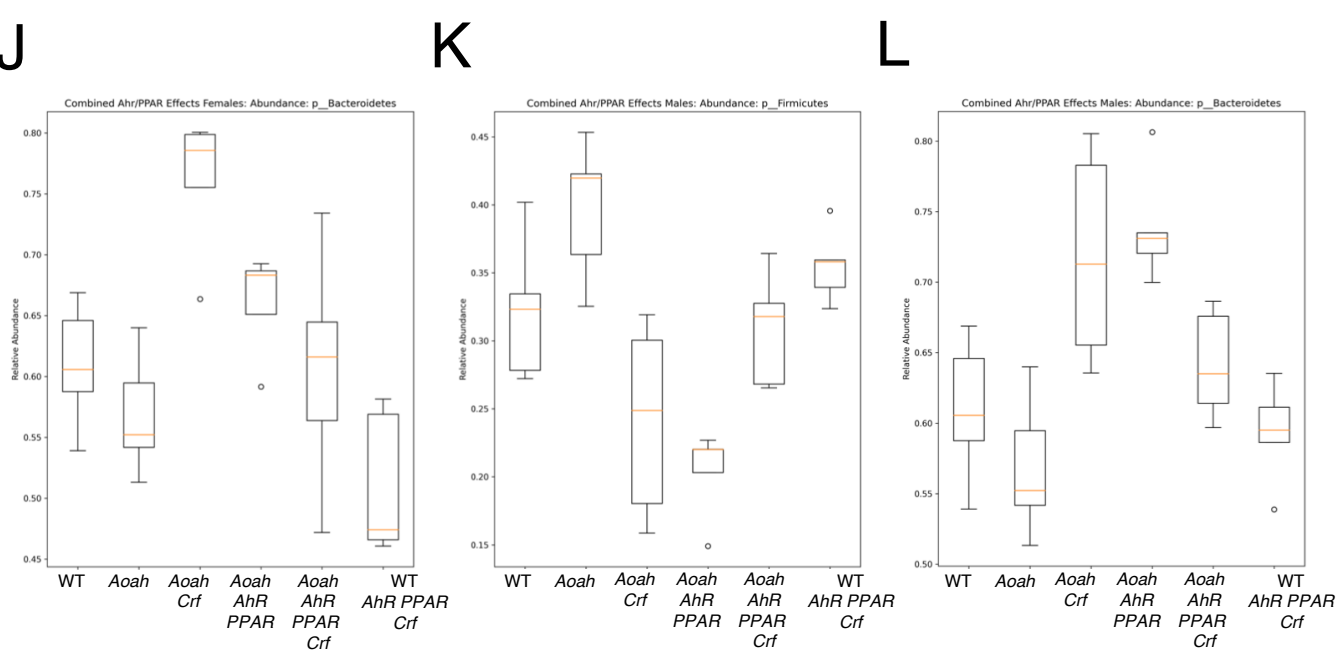